A. Boccuto, Dipartimento di Matematica e Informatica, via Vanvitelli 1, I-06123 Perugia, Italy. e-mail: boccuto@dipmat.unipg.it

B. Riečan, Matematický Ústav, Slovenská Akadémia Vied, Štefánikova 49, SK-81473 Bratislava, Slovakia. e-mail: riecan@mat.savba.sk,

riecan@fpv.umb.sk

\title{
A NOTE ON A PETTIS-KURZWEIL-HENSTOCK TYPE INTEGRAL IN RIESZ SPACES
}

\begin{abstract}
Recently a connection has been found between the improper KurzweilHenstock integral on the real line and the integral over a compact space. In this paper these results are extended to a Pettis-type integral for the case of functions with values in Riesz spaces with "enough" order continuous functionals.
\end{abstract}

\section{Introduction.}

In [12] two possibilities are mentioned for defining the improper KurzweilHenstock integral on the real line. Their coincidence has been proved in [6]. On the other hand in [1] and [13] the Kurzweil-Henstock integral has been studied for real functions defined on a compact space. In [5] a natural connection was established between these two situations: the improper integral on the real line and the integral on a compact space. Here we introduce and investigate a Pettis-type integral (p-integral) for functions with values in a Dedekind complete Riesz space $R$ such that the space $R^{*}$ of its order continuous functionals separates the points of $R$, and we shall show that the above mentioned relation holds even for the p-integral. Furthermore, some convergence-type theorems are proved.

Key Words: Riesz spaces, compact topological spaces, order continuous linear functionals, Henstock-Kurzweil integral, Pettis integral.

Mathematical Reviews subject classification: 28B15, 28B05, 28B10, 46G10.

Received by the editors July 29,2002

* This work is supported by CNR (Italian Council of the Researches) and SAV (Slovak Academy of the Sciences). 


\section{Preliminaries.}

Let $\mathbb{N}$ be the set of all strictly positive integers, $\mathbb{R}$ be the set of the real numbers and $\mathbb{R}^{+}$be the set of all strictly positive real numbers. We begin with some preliminary definitions and results.

Definition 2.1. A Riesz space $R$ is said to be Dedekind complete if every nonempty subset of $R$, bounded from above, has supremum in $R$.

Definition 2.2. Let $R$ be a Dedekind complete Riesz space. A sequence $\left(r_{n}\right)_{n}$ of elements of $R$ is said to be bounded if there exist $s_{1}, s_{2} \in R$ such that $s_{1} \leq r_{n} \leq s_{2}$ for all $n \in \mathbb{N}$. Analogously we can define boundedness of a net $\left(r_{\beta}\right)_{\beta \in \Lambda}$ of elements of $R$, where $(\Lambda, \geq) \neq \emptyset$ is a directed set. Given a bounded sequence $\left(r_{n}\right)_{n}$ in $R$, we define

$$
\limsup _{n} r_{n}=\inf _{n}\left[\sup _{m \geq n} r_{m}\right] \text { and } \liminf _{n} r_{n}=\sup _{n}\left[\inf _{m \geq n} r_{m}\right] .
$$

Given a net $\left(r_{\beta}\right)_{\beta}$ in $R$, let

$$
\limsup _{\beta} r_{\beta}=\inf _{\beta}\left[\sup _{\alpha \geq \beta} r_{\alpha}\right] \text { and } \liminf _{\beta} r_{\beta}=\sup _{\beta}\left[\inf _{\alpha \geq \beta} r_{\alpha}\right],
$$

provided that these quantities exist in $R$. We say that $\left(r_{\beta}\right)_{\beta}$ order converges (or simply (o)-converges ) to $r \in R$ if $r=\lim \sup _{\beta} r_{\beta}=\liminf _{\beta} r_{\beta}$, and we write $(o)-\lim _{\beta \in \Lambda} r_{\beta}=r$. We say that a bounded sequence $\left(r_{n}\right)_{n}$ in $R$ order converges (or simply (o)-converges) to $r \in R$ if $r=\limsup _{n} r_{n}=\liminf _{n} r_{n}$, and we write $(o)-\lim _{n} r_{n}=r$.

Let $R$ be as above. A linear functional $g: R \rightarrow \mathbb{R}$ is said to be positive if $g(r) \geq 0$ for each $r \in R, r \geq 0$; order continuous, if for every net $\left(r_{\beta}\right)_{\beta}$ in $R$ such that $(o)-\lim _{\beta} r_{\beta}=0$ we have that $\lim _{\beta} g\left(r_{\beta}\right)=0$. We note that a positive functional $g$ is order continuous if and only if $x_{\beta} \downarrow 0$ in $R$ implies $g\left(x_{\beta}\right) \downarrow 0$ in $\mathbb{R}$, and also if and only if $0 \leq x_{\beta} \uparrow x$ in $R$ implies $g\left(x_{\beta}\right) \uparrow g(x)$ in $\mathbb{R}$. The vector space of all order continuous linear functionals on $R$ will be denoted by $R^{*}$. This space is always a Dedekind complete Riesz space (see [3], p. 55). For example, if $1 \leq p<+\infty$ and $1 / p+1 / q=1$, then $l_{p}^{*}=l_{q}$ and $L_{p}([0,1])=L^{q}([0,1])$. We say that $R^{*}$ separates points of $R$ if for every $r \in R$, $r \neq 0$, there exists $g \in R^{*}$ such that $g(x) \neq 0$. From now on we always suppose that $R$ is a Dedekind complete Riesz space, such that $R^{*}$ separates points of $R$. An example of a Riesz space $R$ satisfying this property, though $R^{* *} \neq R$, is the space $c_{0}$ of all sequences of real numbers, convergent to zero (see [8]). Recall that $g_{1} \geq g_{2}$ in $R^{*}$ means $g_{1}(x) \geq g_{2}(x) \forall x \in R, x \geq 0$, and that an element $x \in R$ satisfies $x \geq 0$ if and only if $g(x) \geq 0$ holds for each $0 \leq g \in R^{*}$ 
(see also [3], Theorem 5.1, p.55). For each $x \in R$, an order continuous linear functional $\widehat{x}$ can be defined on $R^{*}$ via the formula $\widehat{x}(f)=f(x), f \in R^{*}$. Thus, a positive operator $x \mapsto \widehat{x}$ can be defined from $R$ into $R^{* *}$. This operator, which we denote by $c$, is called the canonical embedding of $R$ into $R^{* *}$. The map $c$ is one-to-one if and only if $R^{*}$ separate points of $R$. In economic models, a way to describe the commodity-price system is the pair $\left(R, R^{*}\right)$, in which the hypothesis that $R^{*}$ separates points of $R$ is essential (see [2], pp. 100 and $115)$.

\section{The p-integral.}

Let $X$ be a Hausdorff compact topological space. If $A \subset X$, then the interior of the set $A$ is denoted by int $A$.

We shall work with a family $\mathcal{F}$ of compact subsets of $X$ such that $X \in \mathcal{F}$ and closed under intersection and a monotone and additive mapping $\lambda: \mathcal{F} \rightarrow$ $[0,+\infty)$. The additivity means that

$$
\lambda(A \bigcup B)+\lambda(A \bigcap B)=\lambda(A)+\lambda(B)
$$

whenever $A, B, A \cup B \in \mathcal{F}$.

By a partition (in detail, $(\mathcal{F}, \lambda)$-partition ) of a nonempty set $A \in \mathcal{F}$ we mean a finite collection $\Pi=\left\{\left(A_{1}, \xi_{1}\right), \ldots,\left(A_{q}, \xi_{q}\right)\right\}$ such that:

(i) $A_{1}, \ldots, A_{q} \in \mathcal{F}, \bigcup_{i=1}^{q} A_{i}=A$,

(ii) $\lambda\left(A_{i} \bigcap A_{j}\right)=0$ whenever $i \neq j$,

(iii) $\xi_{j} \in A_{j}(j=1, \ldots, q)$.

Sometimes, when no confusion can arise, we will indicate by partition of $A$ a finite collection $\left\{A_{j}: j=1, \ldots, q\right\}$, satisfying conditions (i) and (ii). If $F: \mathcal{F} \rightarrow \mathbb{R}$ is a set function and $\Pi=\left\{A_{j}: j=1, \ldots, q\right\}$ is a partition of $\emptyset \neq A \in \mathcal{F}$, we denote by $\sum_{\Pi} F$ the quantity $\sum_{j=1}^{q} F\left(A_{j}\right)$.

We shall assume that $\mathcal{F}$ separates points in the following way: to any $A \in \mathcal{F}$ there exists a sequence $\left(\mathcal{A}_{n}\right)_{n}$ of partitions of $A$ such that

(i) $\mathcal{A}_{n+1}$ is a refinement of $\mathcal{A}_{n}$,

(ii) for any $x, y \in A, x \neq y$, there exist $n \in \mathbb{N}$ and $B \in \mathcal{A}_{n}$ such that $x \in B$ and $y \notin B$. 
We note that this assumption is fulfilled if $\mathcal{F}$ consists of all compact sets and the topological space $X$ is metrizable or it satisfies the second axiom of countability (see [13]).

A gauge on a set $A \in \mathcal{F}$ is a mapping $\delta$ assigning to every point $x \in A$ a neighborhood $\delta(x)$ of $x$. We endow the set of all gauges on $A$ with the order

$$
\delta_{1} \leq \delta_{2} \Longleftrightarrow \delta_{1}(x) \subset \delta_{2}(x) \quad \forall x \in A .
$$

If $\Pi=\left\{\left(A_{1}, \xi_{1}\right), \ldots,\left(A_{q}, \xi_{q}\right)\right\}$ is a partition of $A$ and $\delta$ is a gauge on $A$, then we say that $\Pi$ is $\delta$-fine if $A_{j} \subset \delta\left(\xi_{j}\right)$ for any $j=1,2, \ldots, q$.

We obtain a simple example putting $X=[a, b] \subset \mathbb{R}$ with the usual topology, $\mathcal{F}=$ the family of all closed subintervals of $X, \lambda([\alpha, \beta])=\beta-\alpha, a \leq \alpha<$ $\beta \leq b$. Any gauge can be represented by a real function $d:[a, b] \rightarrow \mathbb{R}^{+}$, if we put $\delta(x)=(x-d(x), x+d(x))$.

Another example is the unbounded interval $[a,+\infty]=[a,+\infty) \bigcup\{+\infty\}$ considered as the one-point compactification of the locally compact space $[a,+\infty)$. The base of open sets consists of open subsets of $[a,+\infty)$ and the sets of the type $(b,+\infty) \bigcup\{+\infty\}, a \leq b<+\infty$. Any gauge in $[a,+\infty]$ has the form $\delta(x)=(x-d(x), x+d(x))$, if $x \in[a,+\infty] \cap \mathbb{R}$, and $\delta(+\infty)=(b,+\infty]=$ $(b,+\infty) \bigcup\{+\infty\}$, where $d$ denotes a positive real-valued function defined on $[a,+\infty)$, and $b$ denotes a real number, with $b \geq a$.

We now define the p-integral on $X$. If $\Pi=\left\{\left(A_{1}, \xi_{1}\right), \ldots,\left(A_{q}, \xi_{q}\right)\right\}$ is a partition of a set $A \in \mathcal{F}$, and $f: X \rightarrow R$, then we define the Riemann sum by

$$
S(f, \Pi)=\sum_{j=1}^{q} \lambda\left(A_{j}\right) f\left(\xi_{j}\right) .
$$

We note that the fact that $\mathcal{F}$ separates points guarantees the existence of at least one $\delta$-fine partition for any gauge $\delta$ (see [13], [19]).

Definition 3.1. A function $f: X \rightarrow R$ is p-integrable on a set $A \in \mathcal{F}$, if there exists $I \in R$ such that $\forall \varepsilon>0$ and $\forall g \in R^{*}$ there exists a gauge $\delta$ on $A$ such that

$$
|g(S(f, \Pi))-g(I)| \leq \varepsilon
$$

whenever $\Pi$ is a $\delta$-fine partition of $A$. We denote $I$ by $\int_{A} f$.

Remark 3.2. We note that, if $I_{1}, I_{2} \in R$ satisfy (1), then $I_{1}=I_{2}$. Indeed, for all $g \in R^{*}, g \geq 0$, and for large enough partition $\Pi$, we get

$$
\left|g\left(I_{1}\right)-g\left(I_{2}\right)\right| \leq\left|g\left(I_{1}\right)-g(S(f, \Pi))\right|+\left|g(S(f, \Pi))-g\left(I_{2}\right)\right| \leq 2 \varepsilon .
$$

Since $R^{*}$ separates the points of $R$, then we get $I_{1}-I_{2}=0$, that is the assertion. 
Remark 3.3. It is easy to check that, in the case $R=\mathbb{R}$, the p-integral coincides with the classical Kurzweil-Henstock integral, as introduced in [5]. In this case, we often will use the term "integrable" instead of "p-integrable".

We now state the main properties of the p-integral.

Proposition 3.4. If $f_{1}, f_{2}$ are p-integrable on $A \in \mathcal{F}$ and $c_{1}, c_{2} \in \mathbb{R}$, then $c_{1} f_{1}+c_{2} f_{2}$ is p-integrable on $A$ and

$$
\int_{A}\left(c_{1} f_{1}+c_{2} f_{2}\right)=c_{1} \int_{A} f_{1}+c_{2} \int_{A} f_{2} .
$$

The proof is similar to the one of [12], Theorems 2.5.1 and 2.5.3.

Proposition 3.5. If $f: X \rightarrow R$ is p-integrable on $A \in \mathcal{F}$, then for every $g \in R^{*}$ the real-valued map $g \circ f$ is integrable on $\mathcal{A}$, and

$$
\int_{A} g \circ f=g\left(\int_{A} f\right)
$$

Conversely, if $f: X \rightarrow R$ is such that $g \circ f$ is integrable on $A \in \mathcal{F}$ for each $g \in R^{*}$ and there exists $I \in R$ such that

$$
\int_{A} g \circ f=g(I) \quad \forall g \in R^{*}
$$

then $f$ is p-integrable on $A$, and $\int_{A} f=I$.

Proof. Fix an arbitrary $g \in R^{*}$ and a partition $\Pi$ of $A, \Pi=\left\{\left(A_{i}, \xi_{i}\right): i=\right.$ $1, \ldots, q\}$. We have

$$
\begin{aligned}
g(S(f, \Pi)) & =g\left(\sum_{i=1}^{q} \lambda\left(A_{i}\right) f\left(\xi_{i}\right)\right) \\
& =\sum_{i=1}^{q} \lambda\left(A_{i}\right) g\left(f\left(\xi_{i}\right)\right)=S(g \circ f, \Pi) .
\end{aligned}
$$

The assertion follows from (3) and definitions of integrability and p-integrability.

Proposition 3.6. If $f_{1}$ and $f_{2}$ are $p$-integrable on $A \in \mathcal{F}$ and $f_{1} \leq f_{2}$, then $\int_{A} f_{1} \leq \int_{A} f_{2}$. 
Proof. Fix arbitrarily $g \in R^{*}, g \geq 0$. Then $g \circ f_{1} \leq g \circ f_{2}$. By the first part of Proposition 3.5 and Proposition 1.4 of [13] we get that $g \circ f_{1}$ and $g \circ f_{2}$ are integrable on $A$, and

$$
\int_{A} g \circ f_{1} \leq \int_{A} g \circ f_{2} .
$$

Again by Proposition 3.5, we have

$$
\int_{A} g \circ f_{l}=g\left(\int_{A} f_{l}\right), \quad l=1,2 .
$$

From (4) and (5) it follows that

$$
g\left(\int_{A} f_{1}\right) \leq g\left(\int_{A} f_{2}\right) .
$$

The assertion follows from (6) and arbitrariness of $g \in R^{*}$.

A simple consequence of Proposition 3.6 is the following assertion.

Corollary 3.7. If both $f$ and $|f|$ are p-integrable on $A \in \mathcal{F}$, then

$$
\left|\int_{A} f\right| \leq \int_{A}|f|
$$

We now state the following results.

Proposition 3.8. Let $u \in R, u \geq 0$. For every $E \in \mathcal{F}$, the function $f=$ $\chi_{E} u: X \rightarrow R$ satisfies the condition

$$
\exists I \in R \text { such that } \forall \varepsilon>0, \exists \text { gauge } \delta \text { such that }|S(f, \Pi)-I| \leq \varepsilon u
$$

for all $\delta$-fine partition $\Pi$ of $X$.

Proof. It is enough to apply Proposition 1.5., pp. 155-156, of [13], and to use the same technique as in Theorem 3.18 of [6].

Proposition 3.9. Let $f: X \rightarrow R$ satisfy condition (7) for suitable $I$ and $u \in R, u \geq 0$. Then $f$ is p-integrable on $X$, and $\int_{X} f=I$.

Proof. Let $I$ and $u$ be as in the hypothesis of the proposition. Fix an arbitrary $\varepsilon>0$ and $g \in R^{*}, g \geq 0$. Then there exists $\eta>0$ such that

$$
\eta g(u) \leq \varepsilon
$$


Moreover, by condition (7), in correspondence with $\eta$ there exists a gauge $\delta$ such that

$$
|S(f, \Pi)-I| \leq \eta u
$$

for all $\delta$-fine partitions $\Pi$ of $X$. From (8) and (9) it follows that

$$
|g(S(f, \Pi))-g(I)| \leq g(\eta u)=\eta g(u) \leq \varepsilon
$$

for all $\delta$-fine partitions $\Pi$ of $X$. The assertion follows from (10).

Proposition 3.10. For every $E \in \mathcal{F}$ and $u \in R$ the function $\chi_{E} u$ is $p$ integrable on $X$ and $\int_{X} \chi_{E} u=\lambda(E) u$.

Proof. Since $R$ is a Riesz space, we have $u=u^{+}-u^{-}$, where $u^{+}, u^{-} \in R$, $u^{+} \geq 0, u^{-} \geq 0$. So, we can suppose, without loss of generality, that $u \geq 0$. The assertion follows from Propositions 3.8 and 3.9.

\section{Convergence Theorems}

The following theorem generalizes to the context of Riesz spaces and our Pettistype integral Theorem 3.1 of [5], which was formulated for real-valued functions.

Theorem 4.1. Let $X=X_{0} \bigcup\left\{x_{0}\right\}$ be the one-point compactification of a locally compact space $X_{0}$. Let $f: X \rightarrow R$ be a function such that $f\left(x_{0}\right)=0$. Let $\left(A_{n}\right)_{n}$ be a sequence of sets, such that $A_{n} \in \mathcal{F}, A_{n} \subset \operatorname{int} A_{n+1}, A_{n+1} \backslash$ $\operatorname{int} A_{n} \in \mathcal{F}, \lambda\left(A_{n} \backslash \operatorname{int} A_{n}\right)=0(n=1,2, \ldots), \bigcup_{n=1}^{\infty} A_{n}=X_{0}$. Let $f$ be $p$ integrable on $A_{n}(n=1,2, \ldots)$ and let there exist in $R$ an element $I$ such that, $\forall \varepsilon>0, \forall g \in R^{*}$, there exists an integer $n_{0}$ such that

$$
\left|\int_{A} g \circ f-g(I)\right| \leq \varepsilon \quad \forall A \in \mathcal{F}, X_{0} \supset A \supset A_{n_{0}} .
$$

Then $f$ is $p$-integrable on $X$ and $\int_{X} f=I$.

Proof. By hypothesis and the first part of Proposition 3.5, we get that $g \circ f$ is integrable on $A_{n}$ for all $g \in R^{*}$. Moreover, by Theorem 3.1 of [5], $g \circ f$ is integrable on $X$ and

$$
\int_{X} g \circ f=g(I)
$$


The assertion follows by (11) and the second part of Proposition 3.5.

We now state a monotone convergence Levi-type theorem.

Theorem 4.2. Let $f_{n}: X \rightarrow R, n \in \mathbb{N}$ be p-integrable, $\left(\int_{X} f_{n}\right)_{n}$ be bounded, and suppose that for every $g \in R, g \geq 0$, and $\forall x \in X, g\left(f_{n}(x)\right) \uparrow g(f(x))$. Then $f$ is p-integrable and $\sup _{n} \int_{X} f_{n}=\int_{X} f$.

Proof. Fix an arbitrary $g \in R^{*}, g \geq 0$. By hypothesis, we get that the sequence $\left(g\left(\int_{X} f_{n}\right)\right)_{n}$ is bounded. Thus, by [13], Theorem 2.2, pp. 159162 and the first part of Proposition 3.5, the real-valued function $g \circ f$ is integrable and

$$
\begin{aligned}
\int_{X} g \circ f & =\lim _{n} \int_{X} g \circ f_{n}=\sup _{n} \int_{X} g \circ f_{n} \\
& =\sup _{n}\left[g\left(\int_{X} f_{n}\right)\right]=g\left(\sup _{n} \int_{X} f_{n}\right) .
\end{aligned}
$$

The assertion follows from (12) and the second part of Proposition 3.5.

\section{References}

[1] S. I. Ahmed, W. F. Pfeffer, A Riemann integral in a locally compact Hausdorff space, J. Australian Math. Soc., 41 (1986), 115-137.

[2] Ch. D. Aliprantis, D. J. Brown, O. Burkinshaw, Existence and Optimality of Competitive Equilibria, Springer-Verlag, 1990.

[3] Ch. D. Aliprantis, O. Burkinshaw, Positive Operators, Academic Press Inc., 1985.

[4] A. Boccuto, Differential and integral calculus in Riesz spaces, Tatra Mountains Math. Publ., 14 (1998), 293-323.

[5] A. Boccuto, B. Riečan, A note on the improper Kurzweil-Henstock integral, Acta Univ. Mathaei Belii, 9, (2002), 7-12.

[6] A. Boccuto, B. Riečan, On the Henstock-Kurzweil integral for Riesz-spacevalued functions defined on unbounded intervals, Czech. Math. J., (2002), to appear. 
[7] M. Duchoň, B. Riečan, On the Kurzweil-Stieltjes integral in ordered spaces, Tatra Mountains Math. Publ., 8 (1996), 133-141.

[8] D. H. Fremlin, Topological Riesz Spaces and Measure Theory, Cambridge Univ. Press, 1974.

[9] R. Henstock, The general theory of integration, Clarendon Press, Oxford, 1991.

[10] J. Kurzweil, Nicht absolut-konvergente Integrale, Teubner, Leipzig, 1980.

[11] P. Y. Lee, Lanzhou Lectures on Henstock integration, World Scientific Publishing Co., 1989.

[12] P. Y. Lee, R. Výborný, The integral: An easy approach after Kurzweil and Henstock, Cambridge Univ. Press, 2000.

[13] B. Riečan, On the Kurzweil integral in compact topological spaces, Rad. Mat. 2 (1986), 151-163.

[14] B. Riečan, On the Kurzweil integral for functions with values in ordered spaces, I, Acta Math. Univ. Comenian. 56-57 (1989), 75-83.

[15] B. Riečan, T. Neubrunn, Integral, Measure and Ordering, Kluwer, Dordrecht, and Ister, Bratislava, 1997.

[16] B. Riečan, M. Vrábelová, On the Kurzweil integral for functions with values in ordered spaces, II, Math. Slovaca, 43 (1993), 471-475.

[17] B. Riečan, M. Vrábelová, On integration with respect to operator valued measures in Riesz spaces, Tatra Mountains Math. Publ., 2 (1993), 149165 .

[18] B. Riečan, M. Vrábelová, On the Kurzweil integral for functions with values in ordered spaces, III, Tatra Mountains Math. Publ. 8 (1996), 93-100.

[19] B. Riečan, M. Vrábelová, The Kurzweil construction of an integral in ordered spaces, Czechoslovak Math. J. 48 (123) (1998), 565-574.

[20] C. Wu, Z. Gong, On Henstock integral of fuzzy-number-valued functions (I), Fuzzy sets and systems, 120 (2001), 523-532. 
